\title{
Optoelectronic Properties of Amorphous Silicon the Role of Hydrogen: From Experiment to Modeling
}

\author{
Franco Gaspari \\ University of Ontario Institute of Technology,
}

Canada

\section{Introduction}

Amorphous silicon, and its more useful alloy form, hydrogenated amorphous silicon (a-Si:H), has been the subject of investigation for more than three decades. A-Si:H is a lowcost, efficient material which is used extensively for electronic devices. Indeed, most recent electronic device textbooks contain a comprehensive review of the physics of amorphous materials and amorphous silicon in particular (Baranovski, 2006; Kasap, 2005; Street, 2000). The advantages of a-Si:H are particularly evident when considering the photovoltaic application context for the preparation of solar cells: in fact, a-Si:H has a large optical absorption coefficient (about 0.5 micron of the material will absorb $90 \%$ of the incident sunlight); the energy gap can be modulated to allow for near optimum conversion efficiency for sunlight; it can be alloyed with other elements (carbon, germanium) to create multijunction structures with increased energy conversion efficiency for sunlight. Finally, it is plentiful and can be deposited on a variety of materials (at low temperature, over large areas, and on flexible substrates).

However, the presence of metastable defects in a-Si:H adversely affects the performance of photovoltaic cells and thin film transistors. Electrical conductivity, photoconductivity and luminescence degradation have been linked to defect formation, such as dangling bonds (DBs) in the a-Si:H film (Akkaya \& Aktas, 1995; Street, 1980).

Staebler and Wronski (1977) found that defects can be created by illuminating a-Si:H. The creation of these light-induced defects (LID) is therefore referred to as the Staebler-Wronski (SW) effect. The presence of these defects, or dangling bonds, is the major factor responsible for the deterioration of the optical and electronic properties of a-Si:H. On the other hand, these defects are metastable and can be cured. Indeed, we could define a SW process that can be described as a two-step reversible process:

i. Exposure to sunlight leads to an increase in the density of states (dangling bonds) in the energy gap of a-Si:H; this represents the SW effect proper;

ii. Subsequent annealing at elevated temperatures (150-200 OC) reduces the density of states back to the original value, thus restoring the optoelectronic properties.

It has been shown experimentally that both optical and electronic properties of amorphous silicon, such as refractive index, optical gap, absorption coefficient, electron and hole 
mobility, etc., are strongly dependent on hydrogen content, in terms of both hydrogen concentration and hydrogen dynamics (diffusion) under various conditions - see, for instance, (Searle, 1998) and references therein. The investigation of such dynamics, including the relation with defect creation and annealing, is crucial for assessing the appropriate solutions to achieve better control of the defects and, consequently, better optoelectronic performances.

There exists a large amount of articles and review papers or books that address the basic properties of a-Si:H, including analysis of the structural, optical and electronic properties; description of a variety of experimental methods used for the growth of a-Si:H films; and correlation between growth parameters and film quality.

In this chapter a summary of the basic properties and historical issues related to a-Si:H and its applications in optoelectronics is presented in section 2. A more exhaustive description of the basic properties of a-Si:H is provided by the references in this section. Section 3 will focus on the role of hydrogen in relation to the optoelectronic properties and defect dynamics in a-Si: $\mathrm{H}$, and will examine some of the prominent models of hydrogen diffusion also used to describe the SW process dynamics. Section 4 will describe the use of tritium, an isotope of hydrogen, as an experimental probe that can be used as a reference by such models. Finally, section 5 will present the results of an integrated experimental and theoretical approach aimed at developing a proper model of the dynamics inherent to a-Si:H and amorphous materials in general. Future work necessary to achieve a proper description of these dynamic processes will be indicated in the Conclusion section.

\section{Properties of a-Si:H}

There exist several preparation methods for a-Si:H films. Early work on evaporated and sputtered a-Si:H lead to poor quality films, and it is now widely accepted that Radio Frequency (RF) Glow Discharge produces the best quality material, although other more recent methods claim similar or better results. A comprehensive review of the advantages and disadvantages of the different methods employed to grow a-Si:H can be found in the books edited by Searle (1998) and Street (1991).

In general, it is desirable that a hydrogen plasma be employed to help the formation of $\mathrm{Si}-$ $\mathrm{H}_{\mathrm{n}}$ ion radicals; hence, methods based on plasma-enhanced chemical vapour deposition (PECVD) techniques are usually preferred. The ions produced in the plasma region are directed via an electric field towards a substrate, where film growth takes place. A common characteristic of these PECVD techniques is the possibility of tuning the system using several parameters, which might be mutually dependent on or independent of each other, like partial gas pressure, electrode bias, substrate bias, flow rates, gas mixtures, substrate temperature, and any other adjustable parameter. A review of plasma deposition of a-Si:H can also be found in (Bruno et al.,1995).

If the goal of current research in this sector is the understanding and prediction of the properties of a-S:H, it is crucial that the dependence of physical properties on preparation conditions be fully examined. This requires the development of experimental and predictive tools applicable to size scales ranging from the atomic to the macroscopic levels. Both Searle (1998) and Street (1991) provide an exhaustive review of the structural, optical and electronic properties of a-Si:H, and point out the still unresolved issues. In the following subsection, the basic properties of a-Si:H are presented, with a focus on the role of hydrogen. 


\subsection{Structure and Density of States (DOS)}

In order to understand the implication of the amorphous structure of a-Si:H on its optoelectronic properties, it is useful to examine the structure of amorphous silicon in comparison to its crystalline form (c-Si). Crystalline silicon is characterized by the well known diamond (or tetrahedral) structure, with bond length of $23.3 \mathrm{~nm}$ and bond angle of $109.5^{\circ}$. As a matter of fact, the amorphous form shows very small changes from the crystalline parameters, with a $\pm 10 \%$ deviation in bond length, and a $\pm 5 \%$ deviation in bond angle. These small changes make it possible to maintain a relatively good short range order (within the first 2-3 nearest neighbours); however, the accumulation of structural stress, due to the progressive compounding of small deviations, eventually leads to bond breaking and the appearance of dangling bonds. Figure 1 shows simple 2-d schematics of the formation of dangling bonds: a 2-d square crystal (1a) is slightly distorted ( $1 \mathrm{~b}$, top, center atom). The distortions become more marked as the network is extended, and eventually a dangling bond (DB) appears to relieve the structural stress (top right quadrant of figure 1c: this is usually also accompanied by under-coordinated and over-coordinated bonds).

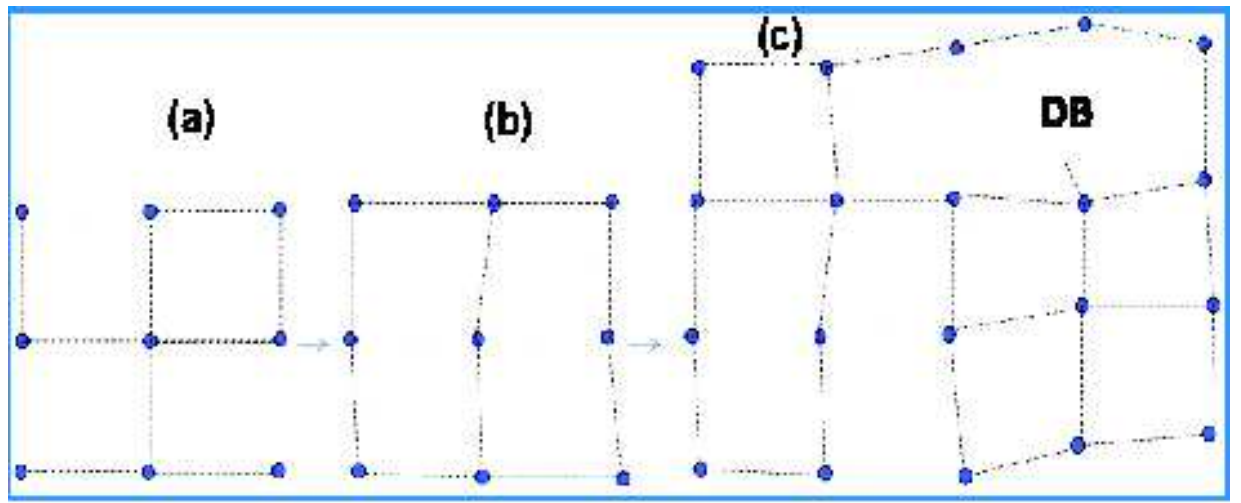

Fig. 1. (a to c) 2-d schematics of formation of dangling bonds due to long range disorder.

The negative effects of the dangling bonds on the opto-electronic properties of a-Si can be effectively removed by hydrogenation; that is, hydrogen atoms are introduced to passivate (bond to) the dangling bonds; see, for instance, (Kasap, 2005; Street 1991, 2000).

Hydrogen atoms incorporated into the films satisfy the covalent bonds at defects and microvoids and also allow the lattice to relax, thereby reducing the density of localized states by several orders of magnitude. Figure 2(b) show a 3-d representation of amorphous silicon with dangling bonds passivated by hydrogen atoms. A crystalline structure is also shown for comparison in figure 2(a).

The differences and similarities between the crystalline silicon and amorphous silicon structures are evident when we examine the radial distribution functions (RDF) for the two structures, as shown in Figure 3. The amorphous structure still shows ordered, crystalline features for the first 3 nearest neighbors. The first neighbor also maintains the crystalline sharpness for the peak, while the progressive deviations from the crystalline structure are evident in the spreading of the peaks for the second and third nearest neighbor. 


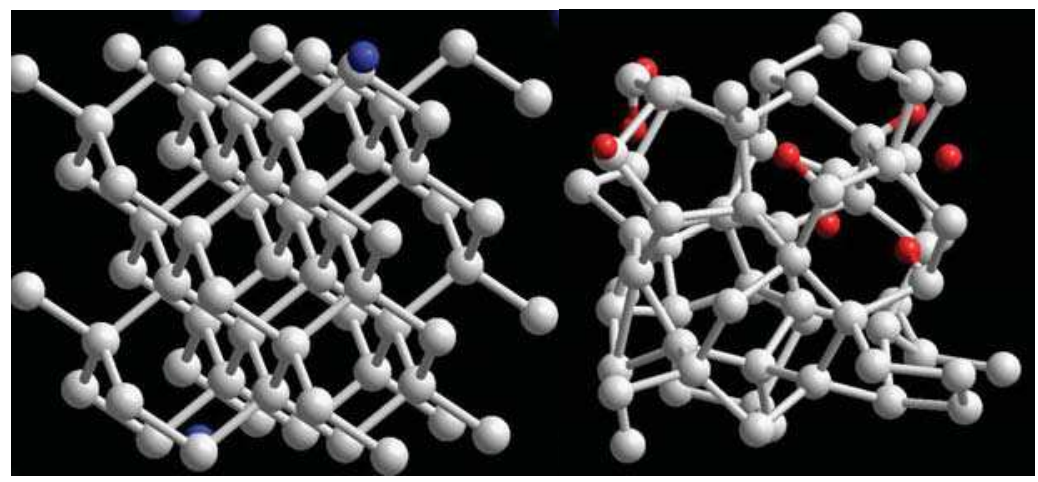

(a)

(b)

Fig. 2. A 3-d computer model representation of c-Si (a), and a-Si (b) with dangling bonds passivated by hydrogen atoms (red balls).

The role of hydrogen in determining the degree of disorder is also the subject of numerous studies. For instance, O'Leary et al. (1996), by using optical absorption data, and by investigating how the modeling parameters vary with the bonded hydrogen concentration, suggest that bonded hydrogen helps decreasing the amount of disorder, and has an impact on the optical absorption spectrum.

More recently, Ukpong (2007) studied the chemically-induced disorder-to-order transition in hydrogenated amorphous silicon as a function of hydrogen concentration, $\mathrm{C}_{\mathrm{H}}$. The author identifies three stages, associated with low $\mathrm{C}_{\mathrm{H}}$, medium $\mathrm{C}_{\mathrm{H}}$, and high $\mathrm{C}_{\mathrm{H}}$, that describe the changes in the stress and structure parameters. Rui et al (2005) investigated the effect of hydrogen plasma annealing on the micro-structural transition from disorder to order in amorphous silicon films. They found that there exist two steps for the reaction between atomic hydrogen and Si network, and show that the hydrogen plasma treatment conditions strongly influence the microstructures of the amorphous Si films

The disorder inherent in the amorphous structure and the presence of dangling bonds has a crucial impact also on the electronic density of states (DOS) of amorphous silicon. Figure 4 shows a simple schematic representation of the electronic DOS of a-Si:H.

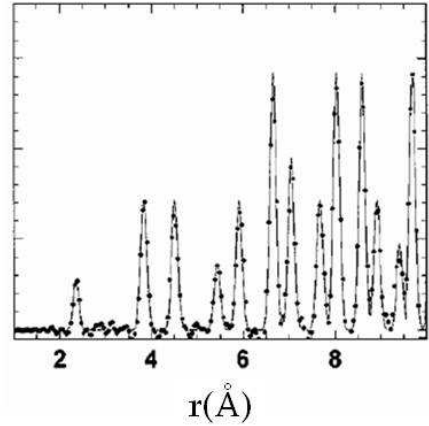

Powdered Crystalline Silicon

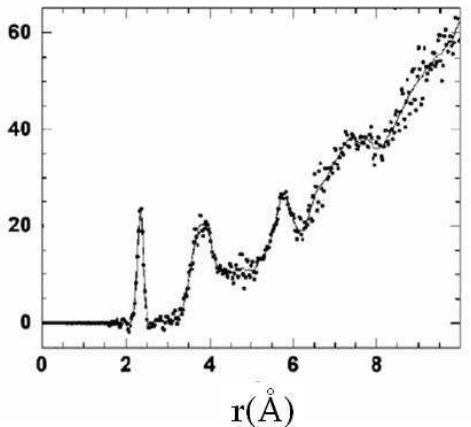

Amorphous Silicon

Fig. 3. Radial Distribution Function of crystalline silicon (left) and amorphous silicon (right) [From: Laaziri et al., 1999] 


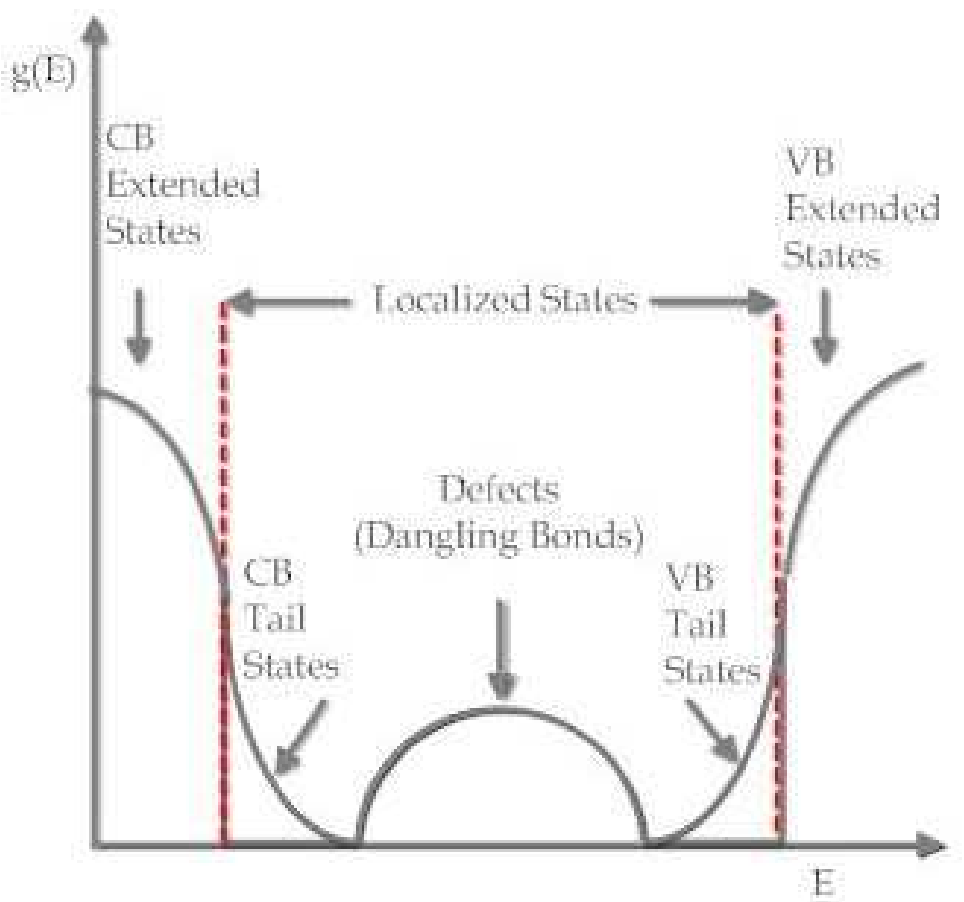

Fig. 4. A schematic representation of the electronic density of states, $g(E)$, of a-Si:H. VB indicates the valence band and CB the conduction band. The dashed, red, vertical lines show the mobility edges, which are defined as the energy level separating extended (nonlocalized) states from localized states.

The main features in Figure 4 can be summarized as follows:

i. The localized tail states are a consequence of the disorder inherent to the amorphous structure. Several studies have examined the role and the extent of tails states in a-Si:H. In particular, a characteristic energy, $E_{\mathrm{U}}$, or Urbach energy, gives the measure of the width of the tail states; hence, it is also referred to as the Urbach tail width - see, for instance, (Ley, 1983). The characteristic width for the band tail states is about $50 \mathrm{meV}$ for the valence band tail states and about $25 \mathrm{meV}$ for the conduction band tail states see, for instance, (Cody, 1981) and the relevant articles cited in the reviews in (Street, 1991) and (Searle, 1998). Furthermore, the tail states width has been associated with a "degree" of disorder, with the implication that the optoelectronic properties of a-Si:H are also dependent on its value.

ii. The localized defect states in the middle of the gap are associated with the formation of DBs. Different models have been proposed to identify the percentage and the energy levels of neutral DBs vs. the positive and negative DBs. Indeed, a DB is identified not only by the fact that the bond is unsatisfied, but also by its net charge, which is determined by the number of electrons sharing the dangling bond, i.e., no electrons imply a positive $\mathrm{DB}\left(\mathrm{D}^{+}\right)$, a single electron makes the bond a neutral one $\left(\mathrm{D}^{0}\right)$, while the presence of two electrons lead to negative DBs (D-). One of the most interesting and 
utilized models, describing the energy distribution of the three types of defects, is the so-called defect-pool model (Powell \& Deane, 1996).

iii. The localized states in the band tails become delocalized at a critical boundary called the mobility edge. A mobility gap is then defined as the energy separation between the two mobility edges of the conduction and valence bands.

\subsection{Optical properties of a-Si:H}

A-Si:H can be described as a direct band-gap semiconductor. The original study of Tauc et al. (1966), in which the distributions of electronic states are assumed to be exactly squareroot in character, terminating abruptly at the respective band edges, leads to a simple analysis of optical absorption and luminescence experiments.

Optical absorption and luminescence occur by transition of electrons and holes between electronic states such as conduction and valence bands, tail states, and gap states. Tauc's relation (Tauc, 1966) describes the dependence of the optical absorption constant, $a$, on the energy gap as:

$$
\mathrm{a} \hbar \omega=\mathrm{B}\left(\hbar \omega-\mathrm{E}_{\mathrm{G}}\right)^{2}
$$

Where $B$ is a constant, $\hbar \omega$ is the photon energy and $E_{G}$ is the optical gap.

The empirical determination of the optical gap $E_{\mathrm{G}}$ can then be achieved by plotting $\sqrt{\mathrm{a} \hbar \omega}$ vs. $\hbar \omega$, which is known as Tauc's plot (a schematic illustration of Tauc's plot is shown in Figure 5).

However, the presence of localized tail states extending from the conduction and valence bands into the energy gap makes the determination of an optical gap unclear. For instance, Malik \& O'Leary (2004) and Thevaril \& O'Leary (2010) have addressed the fact that in amorphous semiconductors considerable deviations from square-root distributions of electronic states occur. They claim that the presence of tail states introduces a corresponding tail in the imaginary part of the dielectric function, $\varepsilon_{2}(\hbar \omega)$, which makes the optical gap difficult to determine, i.e., it introduces a considerable amount of uncertainty into the Tauc optical gap determination procedure.

Nevertheless, it is still a common procedure to determine the optical gap by using Tauc's relation, although two different methods have been used to obtain a value for the gap. The first simply extrapolates the high energy, linear section of the plot of $\sqrt{\mathrm{a} \hbar \omega}$ vs. $\hbar \omega$, and takes the intercept with the x-axis as the value of the optical gap, as shown in Figure 5. The second chooses the photon energy at which the absorption coefficient is equal to $10^{4} \mathrm{~cm}^{-1}$, defined as $E_{04}$, as the optical gap.

The characteristic values for the band gap of a-Si:H determined from Tauc's plot range from $\sim 1.7 \mathrm{eV}$ to $\sim 1.9 \mathrm{eV}$. The variations in gap value are due to preparation conditions, but it is well accepted that the main parameter responsible for the value of the optical gap is the hydrogen content $\left(\mathrm{C}_{\mathrm{H}}\right)$.

Indeed, there are numerous studies that have investigated the dependence of the optical gap and other optical parameters, like absorption coefficient and refractive index, on $\mathrm{C}_{\mathrm{H}}$. Earlier studies can be found in the references in (Searle, 1998) and (Street, 1991). In summary, it has been shown that the optical band-gap of a-Si:H tends to increase with hydrogen content; see also, for instance, (Daouahi et al., 2001; Gaspari et al, 1993). 


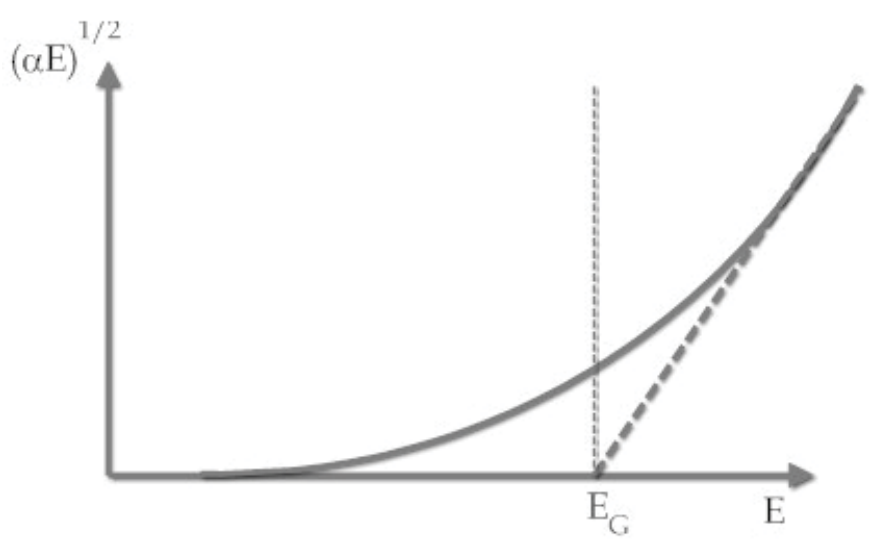

Fig. 5. A schematic illustration of a Tauc's plot. The extrapolation of the high energy linear portion is used to determine the optical gap $\mathrm{E}_{\mathrm{G}}$

\subsection{Electronic properties of a-Si:H}

A-Si:H electronic properties also exhibit a strong dependence on the hydrogen bonding and content. For example, dark conductivity in a-Si:H can be described by two main processes. The first is the standard extended states conduction process, described by the relation (Mott, 1983)

$$
\sigma=\sigma_{0} \exp \left(-E_{\mathrm{A}} / \mathrm{k}_{\mathrm{B}} \mathrm{T}\right)
$$

where $\sigma$ and $\sigma_{0}$ are the electrical conductivity and a prefactor, respectively, and $\mathrm{E}_{\mathrm{A}}, \mathrm{k}_{\mathrm{B}}$ and $\mathrm{T}$ are the activation energy, the Boltzmann constant and the temperature, respectively. $\mathrm{E}_{\mathrm{A}}$ is given by either $E_{C}-E_{F}$ or $E_{F}-E_{V}$, depending on whether electrons or holes are considered, with $E_{C}$ and $E_{V}$ being the conduction band and valence band edges respectively. The second conduction process is referred to as variable-range hopping (VRH) conduction, a well known process in amorphous materials in general. This conduction process is associated with hopping within tail states, and is characterized by the following temperature dependence (Mott, 1983):

$$
\sigma_{h}=\sigma_{h 0} \exp \left(-B / T^{1 / 4}\right)
$$

where $\sigma_{\mathrm{h}}$ and $\sigma_{\mathrm{h} 0}$ are the electrical conductivity and a prefactor, respectively, for variable range hopping.

It has been shown that tail states are also subject to the SW effect (Longeaud et al., 2000). The authors also state that passivation of these DBs in the tail states is related to hydrogen reservoirs. As mentioned before, hydrogen will also influence tail states by reducing the amount of disorder, and by relaxing the structure; furthermore, defects states will tend to shift the Fermi level, thus influencing the activation energy in the dark conductivity. Therefore the hydrogen content plays a fundamental role in determining the conduction processes, as it does for the optical gap. 


\section{The role of hydrogen in a-Si:H}

As previously indicated, the presence of hydrogen during the growth of a-Si:H has a dramatic effect on the optoelectronic properties of a-Si. It is a well established fact that the presence of hydrogen atoms reduces the DB density, both at the center of the gap and in the tail states, thus reducing also the $\mathrm{E}_{\mathrm{U}}$ values. Furthermore, the optical gap increases with hydrogen content. However, several questions are still unanswered: for instance, it is still unclear whether there is a direct relation between hydrogen-content and optical gap, or whether such increase is also due to a structural reordering, leading to a less disordered structure, as indicated by the Urbach width.

More importantly, the role of hydrogen dynamics during the defect creation and defect passivation phases of the SW process is still a matter of debate. The nature of hydrogen bonds, the hydrogen distribution, and hydrogen mobility represent crucial parameters in addressing these issues.

\subsection{Hydrogen bonding}

The most effective characterization of hydrogen content and hydrogen bonding is provided by the vibrational density of states (VDOS), obtained experimentally via transmission and Raman infrared spectroscopy. Fourier Transform Infrared Spectroscopy (FTIR) has become in fact one of the routine modes of investigation to determine the quality of the a-Si:H film (Searle, 1998; Street, 1991; and references therein).

Investigations on the correct interpretation of crucial features in the infrared (IR) spectrum, such as the nature of the stretching modes at about $2000 \mathrm{~cm}^{-1}$, the roles of chains and microvoids, the distinction among different poly-hydride bonds - i.e. $\mathrm{Si}-\mathrm{H}_{2}$ vs. $\mathrm{Si}-\mathrm{H}_{3}$ vs. $\left(\mathrm{Si}-\mathrm{H}_{\mathrm{n}}\right)_{\mathrm{m}}$ - became crucial in order to achieve a better understanding of the role of hydrogen atoms both in the determination of the basic film properties (energy gap, Fermi level, etc.) and in the dynamics of creation and annealing of defects.

For instance, early infrared spectroscopy (Jeffrey et al. 1979; Knights \& Lujan, 1979; Zanzucchi et al., 1977), primarily of evaporated and sputtered a-Si:H, associated polyhydride bonding with poor film properties, but Street \& Tsai (1988) and Kato \& Aoki (1985) showed that that was not the case. A model predicting the various modes of vibration for silicon and hydrogen atoms in a-Si:H was developed by Lucovski et al. (1989).

Recently, the correct interpretation of the various modes, in particular the stretching modes between 1950 and $2150 \mathrm{~cm}^{-1}$, has been questioned (Smets \& van de Sanden, 2007); however the frequency assignments by Lucovski still provide an excellent reference for the investigation of a-Si:H.

\subsection{Hydrogen diffusion models}

Several models have been proposed to describe the dynamics of hydrogen diffusion within the amorphous silicon network relative to the Staebler-Wronski effect. Furthermore, many techniques have been employed to generate a realistic computational model of a-Si:H. In particular, molecular dynamics (MD) has become one of the more powerful and frequently used tools for the correlation of the microscopic characteristic of materials with their macroscopic properties, observed experimentally. In order to underline the variety of models and approaches used to analyze hydrogen diffusion, a summary of some of the most 
important models and experimental studies introduced over the past 20 years is presented below. It should be noted that the following summary represents only a fraction of the publications on this subject, and it is not intended to be exhaustive, but rather to provide a sense of the diversity in the approaches to the problem.

Santos et al. (1991, 1993) present first experimental evidence of light-induced hydrogen motion in undoped a-Si:H, obtained from diffusion experiments under illumination. A definite increase in diffusion was observed for the illuminated samples. The authors speculate that the recombination of e-h pairs releases energy and may induce excitation of hydrogen $(\mathrm{H})$ from a $\mathrm{Si}-\mathrm{H}$ bond. Another important conclusion is that there is an electronic nature to hydrogen motion in a-Si:H. Up to at least $275{ }^{\circ} \mathrm{C}$, the $\mathrm{H}$ diffusion is not purely a thermal process but is dominated by the concentration of carriers.

Jackson \& Tsai (1992) consider hydrogen bonding in terms of a density of states. Bonding in a given configuration is equivalent to occupancy of the state. The barriers to configuration changes are equated with the energy required to reach transport energy. The main conclusions are that there is a range of possibilities: one extreme is the case in which hydrogen is predominantly bonded on void surfaces and the transport energy is substantially different in a-Si than in c-Si; the other extreme is that hydrogen predominantly resides in platelets structures and the transport energy is similar to c-Si. The actual case depends on deposition conditions. Also, Jackson et al. (1993) show that, at relatively high hydrogen concentration, hydrogen atoms reside mainly in clusters. The energy of the clusters depends on the number of hydrogen pairs within the cluster. Annealing has the effect to shift the hydrogen in more stable clusters.

Van de Walle \& Street $(1994,1995)$ investigate, using first principle pseudo-potential calculations, the bonding energetics and the diffusion mechanism of $\mathrm{Si}-\mathrm{H}$ bonds in general and in amorphous silicon. The main conclusions are as follows: i- it is favorable for a hydrogen atom to move from a DB site to a bond centered (BC) site (bond-centered between two silicon atoms); ii- not only is this a favorable path, but the energy levels which are introduced into the band-gap open the way for carrier-enhanced dissociation; iii- the main path is that by which $\mathrm{H}$ stays at approximately the same distance from the original silicon atom, i.e., it moves along the direction of its wagging mode into a $\mathrm{BC}$ site. Finally, the motion of hydrogen atoms through a-Si can be described by a diffusion coefficient:

$$
\mathrm{D}_{\mathrm{H}}=\mathrm{D}_{0} \exp \left(-\mathrm{E}_{\mathrm{A}} / \mathrm{kT}\right)
$$

Where $\mathrm{E}_{\mathrm{A}}$ is the activation energy of the diffusion process.

Biswas et al. (1998) agree that $\mathrm{H}$ diffusion involves some type of hydrogen interstitial state, but that the exact nature of the diffusion mechanism is not well identified. They propose a type of $\mathrm{H}$ diffusion motion more consistent with energetics calculations and experimental evidence. The authors use a tight-binding model to calculate the energy to break a $\mathrm{Si}-\mathrm{H}$ bond and place the hydrogen atom at nearby or distant silicon sites. They find that hydrogen is very reactive, and can form a new $\mathrm{Si}-\mathrm{H}$ bond by breaking a $\mathrm{Si}-\mathrm{Si}$ bond. This is represented by:

$$
\mathrm{Si}-\mathrm{H}+\mathrm{Si}_{\mathrm{x}}-\mathrm{Si}_{\mathrm{y}} \rightarrow \mathrm{Si}^{*}+\mathrm{Si}_{\mathrm{x}}-\mathrm{H}+\mathrm{Si}_{\mathrm{y}}{ }^{*}
$$

These configurations typically consist of two DBs $\left(\mathrm{Si}^{*}\right.$ and $\left.\mathrm{Si}_{\mathrm{y}}{ }^{*}\right)$. Remarkably, the energy of reaction is low even when the $\mathrm{Si}-\mathrm{H}$ bond being broken is not a weak one. The authors 
identify a manifold of transport states, which depends on the bond length deviation of $\mathrm{Si}-$ $\mathrm{Si}$ bonds, through the a-Si:H network. They propose that diffusion of hydrogen proceeds by the hydrogen atom breaking and reforming $\mathrm{Si}-\mathrm{Si}$ bonds in the network, with the diffusing hydrogen carrying a transporting DB along. The basic conclusion is that hydrogen motion is very reactive and does not rely on the existing DBs in the network. It is also somewhat different from the hydrogen motion through bond-centered sites, which according to these authors is a less reactive process since it implies that the $\mathrm{Si}-\mathrm{Si}$ bond must stretch outwards to accommodate the hydrogen atom. The calculation of the energy barriers is complex, but the authors set an upper limit of $0.8 \mathrm{eV}$ and a likely value of $0.5 \mathrm{eV}$.

One of the most important and popular models for hydrogen diffusion is the one proposed by Branz (1999) with the Hydrogen Collision Model (HCM). In this model, DBs are created when recombination of light induced carriers stimulates emission of mobile hydrogen from Si-H bonds according to:

$$
\mathrm{Si}-\mathrm{H} \rightarrow \mathrm{DB}+\mathrm{Si}-\mathrm{H} / \mathrm{DB}
$$

The basic process is described by the following steps:

1. The mobile hydrogen atom goes to a $\mathrm{Si}-\mathrm{Si}$ bond

2. The bond is broken, forming a temporary Si-H and a DB

3. The hydrogen atom hops to another $\mathrm{Si}-\mathrm{Si}$ bond, again breaking the bond, while the previous bond reconstructs itself.

4. The mobile hydrogen atom continues to hop (it can be proven that its binding energy to the various bonds it breaks on its way is weaker than regular $\mathrm{Si}-\mathrm{H}$ ).

5. Eventually, the mobile hydrogen atom re-traps to Si-H through one of two mechanisms, described below:

The first is a normal re-trapping to an immobile $\mathrm{DB}$, given by $\mathrm{Si}-\mathrm{H} / \mathrm{DB}+\mathrm{DB} \rightarrow \mathrm{Si}-\mathrm{H}$, that is, the inverse process of eq. [6]. Basically, one can see this phenomenon as an $\mathrm{H}$ jumping to an ordinary DB, or as the formation of a bond between the mobile DB - that accompanies the mobile $\mathrm{H}$ - and the immobile DB. In both cases, no net DB results from the process.

The second mechanism can be described as a re-trapping process of the form $\mathrm{Si}-\mathrm{H} / \mathrm{DB}+\mathrm{Si}$ $\mathrm{H} / \mathrm{DB} \rightarrow \mathrm{M}(\mathrm{Si}-\mathrm{H})_{2}$. This is far less frequent than normal re-trapping. It represents the collision of 2 mobile $\mathrm{H}$ atoms (and their accompanying mobile DBs) that associate into a metastable complex, $\mathrm{M}(\mathrm{Si}-\mathrm{H})_{2}$, containing a pair of $\mathrm{Si}-\mathrm{H}$ bonds in close proximity. The meaning of this process is that there is a net formation of $2 \mathrm{DBs}$ (the ones left behind by the original Si-H bonds), thus resulting in the SW effect.

All this can be summarized by the following reaction:

$$
2 \mathrm{Si}-\mathrm{H} \rightarrow 2 \mathrm{DB}+2 \mathrm{Si}-\mathrm{H} / \mathrm{DB} \rightarrow 2 \mathrm{DB}+\mathrm{M}(\mathrm{Si}-\mathrm{H})_{2}
$$

Experimental studies by Cheong et al. (2000) examine one of the main and most controversial assumptions of the HC model, namely, that the photo-generated mobile hydrogen atoms can move a long distance at room temperature. They devised an experiment to observe hydrogen motion at room temperature, since detection with traditional methods such as IR and deuterium tracing is inadequate. By using the high sensitivity of the Raman spectrum of electro-chromic amorphous tungsten $\left(\mathrm{a}-\mathrm{WO}_{3}\right)$ to hydrogen insertion the authors were able to detect the long-range motion of hydrogen at room temperature. 
The floating bond (FB) model as described, for instance, in (Biswas \& Pan 2003), proposes an alternative explanation for the $\mathrm{H}$ diffusion process. To put it simply, compared with the hydrogen-collision model proposed by Branz, the creation of DBs is mediated by floating bonds rather than hydrogen atoms.

The proponents of the FB model point out that the emission rate of mobile hydrogen should be larger than the creation rate of a pair of $\mathrm{DB}$ and FB and that the mobility of movable hydrogen should be faster than that of FB, leading to the dominance of the Branz mechanism for DB creation. However, one should note that the possibility of the DB creation by the mobile hydrogen in the case of the HC model is very small, but DB-FB pair creation directly leads to the creation of DB.

As mentioned previously, the hydrogen distribution is also an important parameter in determining the dynamics of the SW effect. For instance, Tuttle \& Adams (1997) show that the energetic and properties of $\mathrm{H}$-atoms must be analyzed considering also their phases, i.e., dilute or clustered. According to the authors, the relative ratio of these phases and their distribution has an important role in determining the properties of a-Si:H. This is a fundamental fact that needs to be taken into account, if a model has to be used to simulate processes connected with hydrogen dynamics, including the testing of the models outlined above that have come to prominence as explanations of the Staebler-Wronski effect.

Gaspari et al. (2010) have examined the hydrogen distribution in simulated samples, obtained by ab-initio Molecular Dynamics (AIMD), by examining the $\mathrm{H}-\mathrm{H}$ radial distribution function. It was noted that the H-structure and its distribution within the underlying silicon network is crucial in determining the properties of a-Si:H and for finding whether the sample possesses high quality characteristics for photovoltaic or micro-electronic applications. These findings are in agreement with results reported in (Tuttle \& Adams, 1997), and indicate that the dilute vs. clustered distribution ratio, combined with a proper interatomic distance, plays a major role in determining the properties of a-Si:H.

More recent studies continue to refine old models and propose new ones; however the focus has now shifted on the computational aspect of the modeling and, in particular, the realism of the model structure. This topic will be discussed in section 5 .

In the following section, a unique procedure providing a novel experimental and theoretical analysis regarding dangling bond formation and annealing in a-Si:H is presented. The approach employed is the incorporation of tritium into a-Si:H.

\section{Tritiated amorphous silicon}

In order to shed light on the role of hydrogen in defect dynamics, it would be desirable to be able to control the evolution of DBs and correlate it with hydrogen dynamics. However, a quantitative study in which the density of DBs is changed usually involves changing deposition conditions, or high-temperature annealing, or damaging the material with highenergy particles or light (Danesh et al., 2005; Schneider \& Schröder, 1990; Sholz et al., 1994). These procedures modify, to varying degree, other structural properties of the material, making it difficult to isolate the effect of DBs on the optoelectronic properties of a-Si : $\mathrm{H}$.

An alternative approach to investigating the properties of a-Si:H has been to substitute hydrogen with one if its isotopes, tritium, and use the effects of the radioactive decay process of tritium as a means to follow the dynamics of defect creation and annealing, and their impact on the opto-electronic properties (Costea et al., 2000; Gaspari et al., 2000; Kherani et al., 2008; Kosteski et al., 2000, 2003, 2005; Zukotynski et al., 2002). 


\subsection{Dangling bond formation due to tritium decay}

Tritium decay is described by the formula: $\mathrm{T} \rightarrow{ }^{3} \mathrm{He}^{+}+\beta^{-}+\bar{v}$, where $\beta^{-}$is the beta-particle emitted by the Tritium atom and $\bar{v}$ is an anti-neutrino. The half-life of tritium is 12.3 years and the mean energy of the beta particle is $5.69 \mathrm{keV}$. The bonding of tritium in amorphous semiconductors is stable at room temperature (Sidhu et al., 1999).

Because of the direct-like nature of the energy gap in amorphous semiconductors, radiative recombination of electron-hole pairs is allowed. Furthermore, the nuclear decay process which leads to the emission of a $\beta$ particle from a Si-T site will produce a ${ }^{3} \mathrm{He}$ atom which will diffuse out and leave behind a dangling bond (or recombination center, as shown in Figure 6). This process has led to a novel way of investigating the properties of amorphous silicon and amorphous semiconductors in general; see, for instance, (Kosteski et al., 2000, 2003; Zukotynski et al., 2002).

It should be noted that tritium decay has side effects that might in turn have an impact on DB formation (i.e., He recoil, e-h pair recombination). These issues have been addressed and it was proven that the impact of the secondary effects is negligible. A detailed analysis of the relative impact of electron-hole pair recombination is presented in the following sub-section.
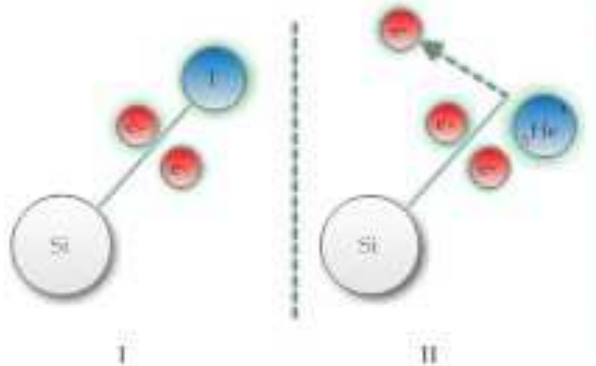

11

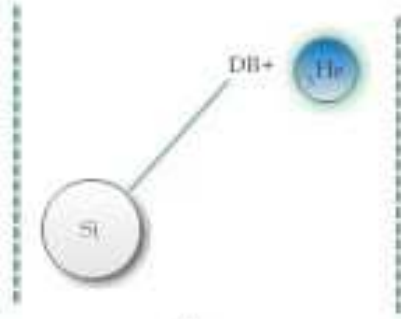

III

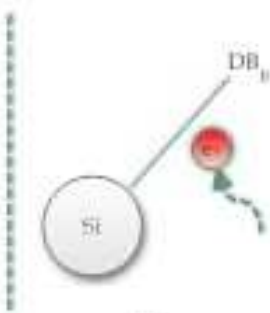

IV

Fig. 6. The mechanism of tritium decay and dangling bond formation: I - Tritium is bonded to a Si atom and shares 2 covalently bonded electrons; II - Tritium decays into ${ }^{3} \mathrm{He}^{+}$and emits a high energy electron ( $\beta$ emission); III - The ${ }^{3} \mathrm{He}^{+}$ion captures both electrons shared with the Si atom and leaves behind a positive dangling bond $\left(\mathrm{DB}^{+}\right)$; IV - The positive $\mathrm{DB}$ can become a neutral DB by capturing in turn an electron generated by collisions between the original $\beta$ particle and the silicon matrix. An average of 1500 electron-hole pairs per $\beta$ emission is generated via this process (Kosteski et al., 2000).

\subsection{Effect of electron-hole pairs on dangling bonds}

The tritium decay process results in the emission of a $\beta$ particle with a mean energy of 5.7 $\mathrm{keV}$. The $\beta$ decay transforms the tritium atom into ${ }^{3} \mathrm{He}^{+}$with a, maximum, recoil energy of about $3 \mathrm{eV}$. Furthermore, electron-hole (e-h) pairs will be created in the material as the $\beta$ particle transfers its energy to the silicon matrix.

It has been shown that electrons with $\mathrm{keV}$ energies are unlikely to produce atomic displacements (Dubeau et al., 1996). They lose energy in collisions with electrons in the material, through which they pass, creating e-h pairs that thermalize by shedding 'Raman' phonons. In the case of a-Si:H, a pair is created for every $4.3 \mathrm{eV}$ of energy lost. It can also be shown that Helium recoil is expected to create little damage (Street et al., 1979; Stutzmann, 1991). 
On the other hand, e-h pairs recombination has also been associated with the creation of DBs (Yelon et al., 2000) and with the mechanism of hydrogen diffusion in a-Si:H (Branz et al., 1993, 1999; Cheong et al., 2000; Santos et al., 1991, 1992). The relative impact of e-h pairs on DB formation can be estimated as follows:

An estimate of the number of e-h pairs generated by tritium decay in an a-Si:H:T film is given by the formula (Kosteski et al., 2000):

$$
\mathrm{G}=\lambda \mathrm{n}_{\mathrm{Tv}} \mathrm{E}_{\mathrm{avg}} / \varepsilon
$$

Where $\lambda$ is the decay constant of tritium $\left(\lambda=1.78 \times 10^{-9} \mathrm{~s}^{-1}\right), \mathrm{E}_{\mathrm{avg}}$ is the average energy of a beta particle $(5.7 \mathrm{keV})$ and $\varepsilon$ is the energy needed to produce an electron-hole pair $(4.3 \mathrm{eV})$; $\mathrm{n}_{\mathrm{Tv}}$ is the volume density of atomic tritium. Assuming $\mathrm{n}_{\mathrm{Tv}} \sim 5$ at. $\%$, as confirmed by IR measurements for standard tritiated samples used in the investigation (Gaspari et al., 2000; Sidhu at al., 1999), we obtain:

$$
\mathrm{G}=5.9 \times 10^{15} \mathrm{~cm}^{-3} \mathrm{~s}^{-1}
$$

The effects of electron beam irradiation on a-Si:H has been extensively investigated (Schneider \& Schroder, 1990; Scholz et al., 1994). In particular, the authors examined the effect of $\mathrm{keV}$ electrons - in the range 1 to $30 \mathrm{keV}$ - and determined that metastable states were formed as a result of the irradiation. The results of their work can be summarized as follows:

- The annealing kinetics of these defects is the same as for those produced by light.

- The DB creation kinetics is linear with time over more than two decades in time.

- The approach to saturation goes from linear to saturated with a change of less than a factor of 2 in the total number of metastable defects.

- There is no sub-linear regime, in contrast to light-induced defect creation and annealing.

- The saturation defect density is independent of dose rate over three orders of magnitude and independent of primary electron energy from less than 1 to more than $30 \mathrm{keV}$.

- Finally, the defect creation rate and saturation are independent of temperature from 170 oC to room temperature.

Yelon et al. (2000) applied the hydrogen collision model developed by Branz to explain the origin of metastable states due to ion bombardment. Since the work by Schroder and coworkers deals in the most part with room temperature $20 \mathrm{keV}$ irradiation, at $170 \mathrm{~mW} \mathrm{~cm}-2$, of samples, $\mathrm{d}=0.6 \mu \mathrm{m}$ thick, the authors have compared their model to the above-mentioned experimental conditions. The intensity of the e-beam corresponds to a flux of $\Phi=5.3 \times 10^{13}$ $\mathrm{cm}^{-2} \mathrm{~s}^{-1}$ electrons striking the sample. In this case, the initial measured creation rate of dangling bonds is $R_{0}=2 \times 10^{15} \mathrm{~cm}^{-3} \mathrm{~s}^{-1}$. They obtained for the volume-averaged pair generation rate the number:

$$
\mathrm{R}=\Phi \mathrm{N}_{\mathrm{p}} / \mathrm{d}=6 \times 10^{20} \mathrm{~cm}^{-3} \mathrm{~s}^{-1}
$$

which is comparable to that of light degradation at the smaller intensities for which smaller $\mathrm{DB}$ creation rates and sub-linear $\left(\mathrm{N}_{\mathrm{db}} \propto \mathrm{t}^{1 / 3}\right)$ kinetics are observed. 
By applying Yelon's treatment to e-h pairs generated by beta particles originating from tritium decay, it can be shown that the number of dangling bond due to e-h recombination is 2 orders of magnitude less than the number due to the direct conversion of a $\mathrm{Si}-\mathrm{T}$ bond into a DB. This is shown in Figure 7.

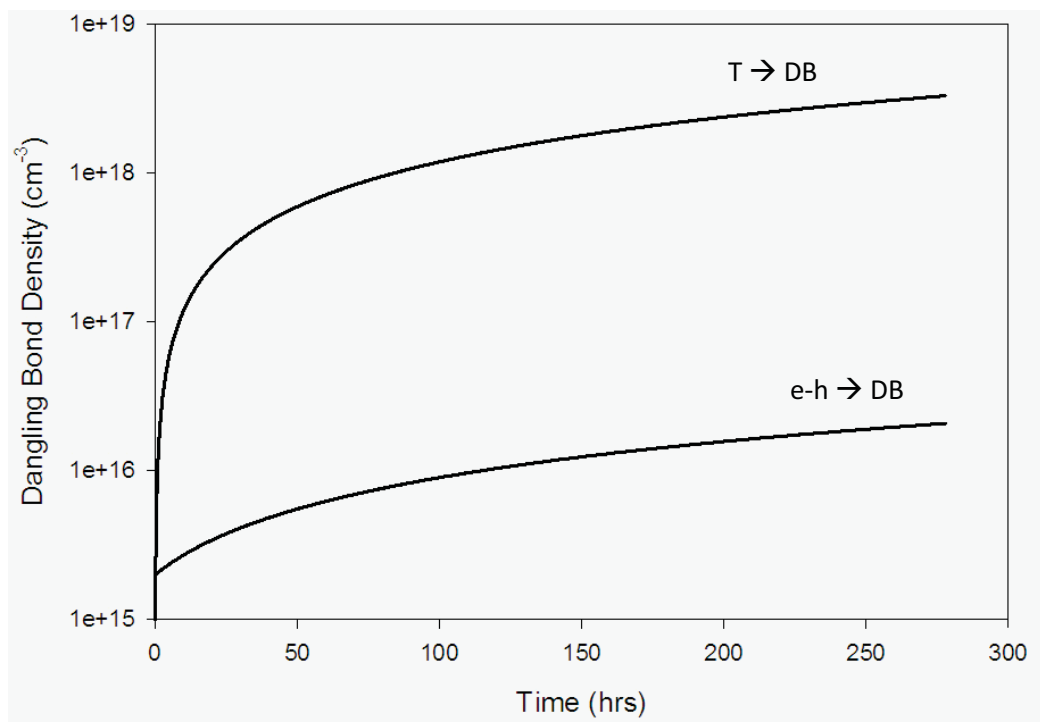

Fig. 7. DB generation rates due to direct conversion of a $\mathrm{Si}-\mathrm{T}$ bond into a $\mathrm{DB}$ and to e-h pair recombination.

In summary, the studies of the properties of a-Si:H:T thin films have established the following:

Creation of DBs, or induced changes to the host structure, by other causes associated with Tritium decay (i.e., irradiation damage from $\beta$ particles, e-h pair recombination, He recoil) is negligible. This is of particular relevance, since it makes it possible to eliminate any side effects of Tritium decay from the analysis, in particular electron irradiation. Calculations have shown that DBs due to electron irradiation caused by Tritium decay account for less than $1 \%$ of the total number of dangling bonds created by the release of the He atom from the Si bond.

Annealing recovers the photoluminescence (PL) and the electrical properties of the tritiated samples (see figures $8 \mathrm{a}$ and $8 \mathrm{~b}$ ). This is the fundamental aspect of the SW process in a-Si:H:T since the dangling bonds are created following a well known nuclear process, in which the Tritium atom is replaced by an Helium atom which diffuses away from the original bond. This clearly shows that the annealing process cannot be a simple reversal of the formation process of DBs. Therefore the passivation of DBs appears to be independent of the DB formation process and linked to the a-Si:H structure and bonding configuration.

The significance of point 2 should be emphasized: many models of the SW effect, in particular earlier ones (Adler, 1984; Stutzmann et al., 1985), are based on the assumption that the annealing mechanism is basically the reverse process of the dangling bond formation mechanism. Although some fundamental common aspect might be present in both phases of the SW process, it is possible to focus on each phase independently. In 
particular, the objective is to establish the relationship between the structure of a-Si:H (i.e. void fraction, hydrogen bonding, etc.) and the curing process (i.e., is it due to $\mathrm{H}$ diffusion and/or a network readjustment during annealing? If $\mathrm{H}$ diffusion is involved, how is it mediated?). A number of fundamental issues remain unresolved: microscopic atom dynamics, for instance, influences atomic structure, chemical bonding, diffusion and vibrations, and are difficult to study both experimentally and theoretically. Furthermore, it is not clear why the number of DBs measured by electron spin resonance (ESR) is one order of magnitude less than expected, as noted by Whitaker et al. (2004) and Ju et al. (2007).

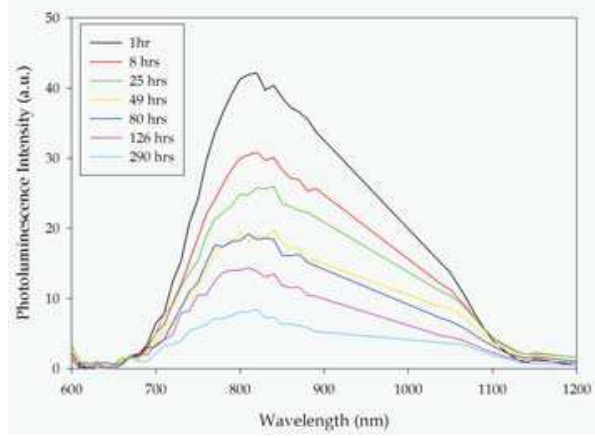

(a)

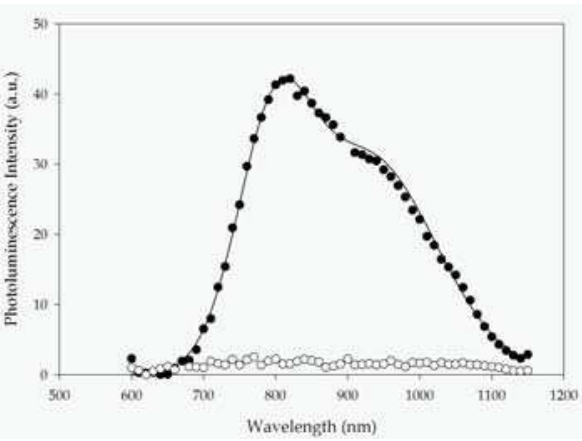

(b)

Fig. 8. (a) Decay of PL signal for a tritiated amorphous silicon film. The black curve was taken one hour after deposition, and the decay vs. time is shown by the coloured curves, labelled in the figure by the post-deposition times at which the spectra were recorded. (b) PL spectrum taken for the same sample after annealing. The open dots show the PL signal (almost completely quenched) approximately two weeks after deposition. The filled circles show the recovered PL signal after annealing at $150 \circ \mathrm{C}$ for one hour.

\subsection{The stretched exponential behaviour}

One of the most interesting observations for tritiated a-Si:H is that the increase in DBs density can be described by a stretched-exponential function, as shown in figure 9 (Kosteski et al., 2003). This indicates that the expected exponential growth of DBs due to tritium decay has a competing process of DB passivation that leads eventually to saturation and a steadystate distribution of DBs.

One hypothesis is that hydrogen atoms that diffuse from nearby sites annihilate the tritium induced defects. In this case, hydrogen detachment from such a site should not be accompanied by the creation of a new defect. This might be possible if the hydrogen atom comes from a large reservoir of paired hydrogen sites such as double-hydrogen complexes (Branz, 1999) or hydrogenated vacancies (Zhang \& Branz, 2001). It should be noted that a similar concept has been proposed for DBs in tail states (Longeau, 2000).

The stretched exponential behaviour has been reported for a number of different conditions for a-Si:H, for instance: Danesh et al. (2005) have investigated a-Si:H degradation for $18 \mathrm{MeV}$ electron beam bombardment. They observed that "the annealing kinetics obeys the stretched-exponential law which is known to be typical for a-Si:H films underwent the lightinduced degradation." 
The stretched exponential behaviour has also been investigated, among others, by Morigaki \& Hikita (2007) and Morigaki et al. (2008) for light-induced defects. Morigaki considers three possible annealing mechanisms, i.e., hydrogen dissociation from two closely located $\mathrm{Si}-\mathrm{H}$ bonds, dissociation from a hydrogen molecule, and reconstruction of silicon network (Zukotynski et al., 2002). Although the dissociation values for the first two processes are higher than the activation energy of thermal annealing $(\sim 1 \mathrm{eV})$, Morigaki argues that the values are still reasonable for activation energies higher than $1 \mathrm{eV}$.

It is not clear, however, why the same stretched exponential behaviour and similar saturation values are observed for tritiated samples, unexposed to light, or for high energy electron bombardment.

The stretched exponential behaviour observed for tritiated amorphous silicon is consistent with the reported values for light induced defects. The major difference between the defect re-equilibration dynamics in a-Si:H:T vs. illuminated a-Si:H is that in the former case there is no assistance from external excitation due to photons.

Yet, it appears that the a-Si:H:T structure still adjusts to the increase in the density of dangling bonds. As previously mentioned, this indicates that a common process, related to a combination of network reconstruction and H-diffusion, should be at the basis of the annealing phase, regardless of the DB formation mechanism.

The hydrogen bonding structure and the hydrogen diffusion process are linked by the activation energies required to bring a hydrogen atom into the mobility band, as indicated, for instance, in (Powell \& Deane, 1996). As the above mentioned authors point out in their "defect pool" model, hydrogen transitions involve silicon DB defects, the defect energy and the Fermi energy. The hydrogen density of states (HDOS) is a crucial factor in this model, and is at the basis of the defect equilibration dynamics.

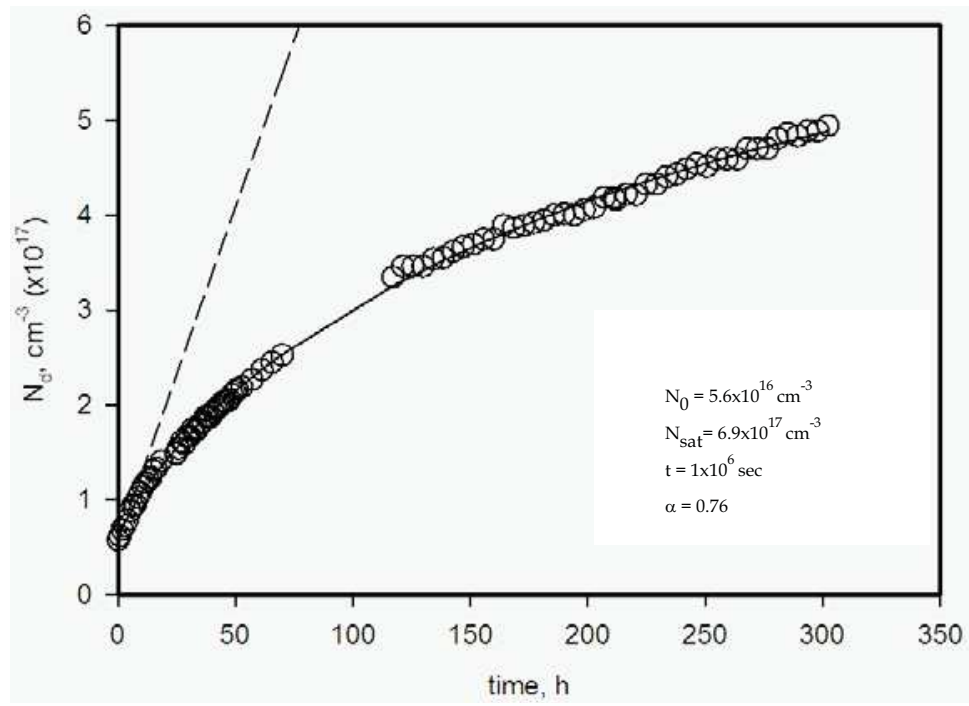

Fig. 9. Dangling bond rate of creation for a-Si:H:T measured by ESR (Kosteski, 2003). The dashed line is the theoretical prediction for one dangling bond per tritium decay. The solid line is a stretched exponential fit to the experimental data. The parameters in the inset refer to the stretched exponential formula: $N_{d}=N_{0}+\left(N_{\text {sat }}-N_{0}\right) \times\left[1-\exp (-t / \tau)^{a}\right]$. 


\section{Ab Initio molecular dynamics}

The main reason for the development of so many models and interpretations of hydrogen mobility is due to the difficulty of obtaining a clear microscopic picture of the dynamic processes via experimental techniques. The only way to investigate the trajectories of hydrogen atoms in a-Si:H is to extensively model it numerically.

Many theoretical techniques have been employed to generate a realistic model of a-Si:H, including classical MD (Ishimaru, 2002; Izumi et al., 2005), tight-binding MD (Singh et al., 2004; Tuttle \& Adams, 1997), and Ab-Initio MD (Su \& Pantelides, 2002). Molecular dynamics has become one of the more powerful and frequently used tools for the correlation of the microscopic characteristic of materials with their macroscopic properties, observed experimentally. Some authors (Abtew \& Drabold, 2006) have applied the model to simulate the dynamics of hydrogen diffusion, in particular as a way of analyzing possible mechanisms behind the Staebler-Wronski (SW) effect. However, classical MD is not sufficiently accurate to describe the covalent bonding and forces in semiconductors, and a tight-binding approach; even DFT-LDA based MD (Singh et al., 2004) is not sufficiently transferable to non-crystalline systems.

For amorphous $\mathrm{Si}$, with no translational symmetry, presence of non-saturated $\mathrm{Si}$ bonds, hydrogen switching between host $\mathrm{Si}$ atoms and diffusion, the most accurate approach is first principles (or ab-initio) MD. Although AIMD has to be used with a smaller unit cell and the simulation time is shorter compared to, for instance, tight-binding, it is extremely accurate in the description of the interatomic potential, forces, and inharmonicity of the interaction.

We have seen that the microscopic details of disordering, hydrogen migration and bonding within the amorphous silicon network are crucial for the understanding of a-Si:H, and for the improvement of the overall quality of the material. However, hydrogen migration and other complex properties must be investigated within a realistic structure to obtain useful information. Kupchak et al. (2008) and Gaspari et al. (2009) have shown that the commonly used radial distribution function (RDF) is not a sufficient validation parameter, and that the recreation of physically consistent vibrational spectra does, on the other hand, represent a sound validation protocol.

The authors have used AIMD to model bulk a-Si:H under various conditions. At various key points in simulations, detailed pictures of measurable quantities related to the electronic structure have been calculated, assuring that the model remain close to nature. In AIMD one treats atoms classically, but the potential is calculated quantum mechanically using DFT. The solutions to the Schrödinger equation are approximated numerically. In terms of computing operations, these calculations involve mainly matrix operations and fast Fourier transforms (FFT). Details of this approach can be found in (Gaspari et al., 2009; Kupchak et al., 2008).

This approach has led to reproducing all the fundamental vibrational signals in a-Si:H. Computer visualization was used to demonstrate some of the most important features, like the existence of poly-hydride bonding. The vibrational spectra obtained for the "good" samples were consistent with experimental data and showed also time stability, as shown in Figures 10 and 11 .

As previously mentioned, Gaspari et al. (2009) have examined the appropriateness of using $\mathrm{RDF}$ as a validation parameter for assessing the realism of a simulated structure. The authors compared a variety of a-Si:H samples with $\mathrm{H}$ content from 0 to $20 \%$, and have found 
that the RDF practically does not depend on the amount of hydrogen in the sample. Furthermore, all the calculated RDF agree reasonably well with the most recent and accurate RDF measurement for a-Si with no hydrogen. This reflects the fact that the most probable distance between neighboring atoms is equal to a sum of the atoms' covalent radii. Even when hydrogen passivates the dangling bonds, this does not modify the Si-Si bond length. On the other hand, atomic vibrations do depend on microscopic bonding (bonds), their angular distribution, distortion or breaking. In fact, the experimental measurements demonstrate a variety of spectral features that obviously require microscopic theoretical interpretation.

Furthermore, in order to further verify the validity of the model, the authors have also studied the special case of metastable Si-H-Si bonds, observed experimentally by Darwich et al. (1995), and have confirmed Darwich's claim within experimental error. Gaspari et al. (2009) indicate that the decrease in the vibrational frequency with respect to that of a stable mono-hydride bond is due to the sharing of the hydrogen electron density between two Si atoms. This decreases the $\mathrm{Si}-\mathrm{H}$ bond strength, increases the bond length and results in reduction of the vibrational frequency. Therefore, the band in the $1500-1800 \mathrm{~cm}^{-1}$ region can be interpreted as the signature of hydrogen metastable bonds, including the TCB bond, with variations in the frequency due to the different overlap between the $\mathrm{H}$ and the Si electron wave functions.

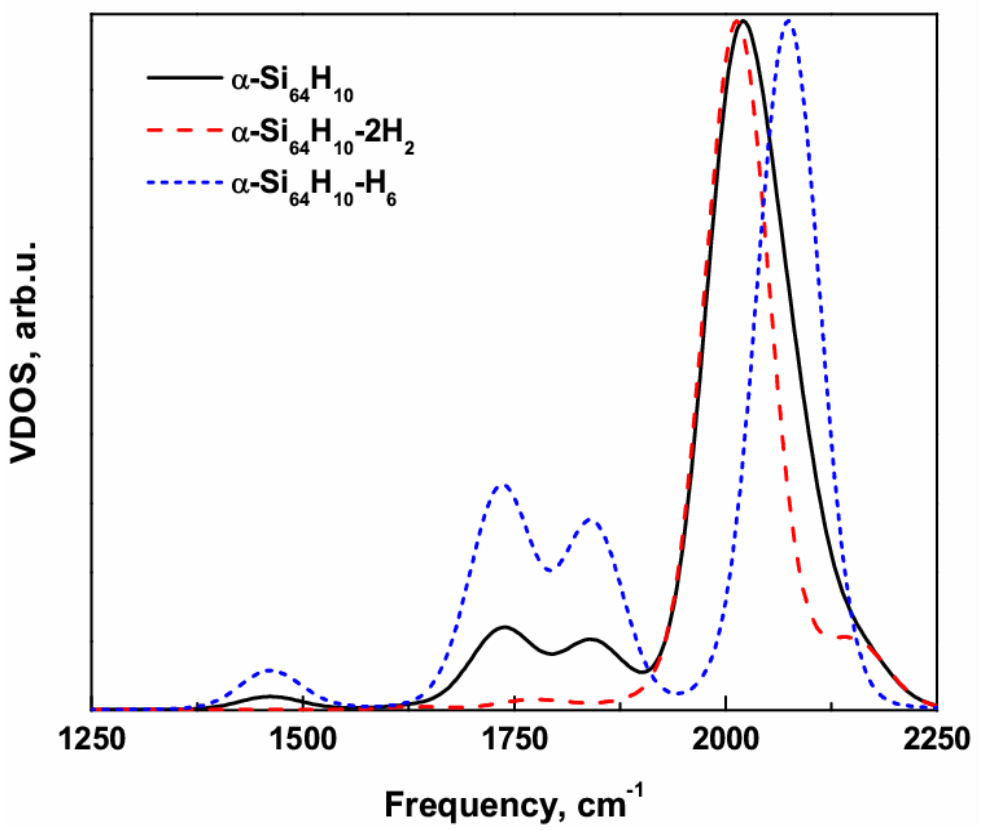

Fig. 10. Hydrogen stretch vibrations for a-Si64-H10 system at high frequency (Kupchak et al., 2008). The solid black line shows all $\mathrm{H}$-associated stretching vibrations, including dihydride modes (blue, short dash) and monohydride modes (red, long dash). Note the very close agreement with data by Lucovsky et al. (1989). 


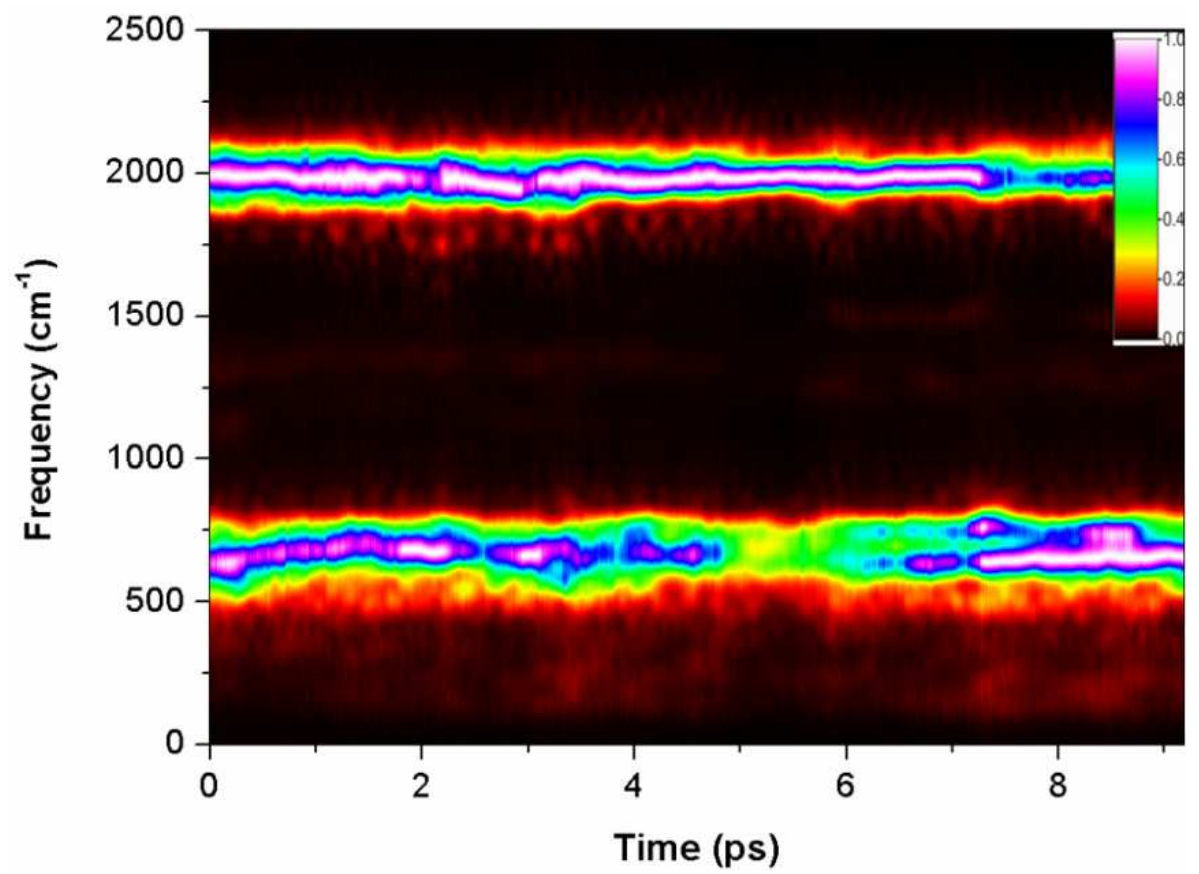

Fig. 11. Time dependent frequencies for a "good" sample. Note the absence of vibrations between the two main modes $\left(2000 \mathrm{~cm}^{-1}\right.$ and $\left.640 \mathrm{~cm}^{-1}\right)$ indicating stability of the bonds. The colour scale is related to the peak intensity, that is, white represents the strongest signal (peak), while black represents no vibrational signal (Kupchak et al., 2008).

The investigation led by this author has proven that in order to validate the simulation of complex structure, bonding, and diffusion, a protocol needs to be established for the verification of the "realism" of the simulated models. Using hydrogenated amorphous silicon as an example, Gaspari et al. $(2009,2010)$ have unambiguously demonstrated that reproduction of the radial distribution function, used commonly in numerical simulations, is not sufficient and must be complemented with verification of other, more complex, macroscopic properties. By focusing on the vibrational modes of the amorphous system, it was proven that the vibrational spectra represent a crucial testing tool for non-crystalline materials because of their complexity and sensitive link to structure and bonding configuration. Successful reproduction of all the experimentally observed vibrational features for a-Si:H has proven the validity of the algorithm and indicates that hydrogen structure and dynamics are extremely sensitive to the parameters of the model. In order to correctly apply a numerical model to extract such important macroscopic parameters as density of states, optical gaps, and migration dynamics, the accuracy should be verified first by the derivation of the standard vibrational modes and comparison with experimental observation.

Indeed, the importance of hydrogen distribution and its connection to hydrogen mobility is demonstrated by recent investigations, both experimental and theoretical, on the role of hydrogen in a-Si:H. For instance, Fehr et al. (2010) investigated the distribution of hydrogen 
atoms around native dangling bonds in a-Si:H by electron-nuclear double resonance (ENDOR). The authors suggest that the hydrogen distribution is continuous and homogeneous and there is no indication for a short-range order between hydrogen atoms and dangling bonds. This is in contrast with current understanding that hydrogen is distributed as a succession of clustered and diluted phases (Gaspari et al., 2010; Tuttle \& Adams, 1997). Such controversies can only be addressed by using a rigorous, realistic model to simulate properties and dynamic processes.

\section{Conclusions}

Hydrogenated Amorphous Silicon (a-Si:H) has been the subject of intensive investigation for over 30 years. The main role of hydrogen in amorphous silicon is the passivation of the Si dangling bonds (DBs) to restore a proper energy gap and the semiconducting properties, thus enabling extensive application of a-Si:H in the microelectronics and the photovoltaic industry. Due to the importance of hydrogen, many experimental methods have been used to characterize the DBs passivation, bonding chemistry and related mechanisms of degradation of the material. Among the numerous experimental techniques used to study aSi:H and the role of hydrogen, the Fourier Transform Infrared Spectroscopy (FTIR) is used extensively to analyze vibrational spectra of a-Si:H. Although FTIR represents one of the most common and powerful techniques, no microscopic links between the observed vibrational features of the hydrogen and the microscopic properties of a-Si:H can be yet established by any experimental means.

A number of other important fundamental issues remain unresolved for a-Si:H as well. Microscopic atom dynamics, for instance, influences atomic structure, chemical bonding, diffusion and vibrations, and are difficult to study both experimentally and theoretically. However, the microscopic details of disordering, hydrogen migration and bonding within the amorphous silicon network is crucial for the understanding of a-Si:H, and for the improvement of the overall quality of the material.

The Staebler-Wronski effect epitomizes this need. It is generally accepted that a-Si:H lightsoaking degradation, observed by Staebler and Wronski, is caused by $\mathrm{Si}-\mathrm{H}$ bonds breaking during illumination. However, the microscopic details of the SW effect are still controversial and it is not clear how to experimentally predict the stability of a-Si:H films, grown at particular temperature and hydrogen concentration, with respect to light induced degradation. Furthermore, a number of alternative techniques have been used to create dangling bonds, and the same dynamics has been observed in the curing (annealing) phase. That is, no matter how the dangling bonds were formed, a similar curing process occurs during annealing. This might be due to diffusion of hydrogen atoms, structural readjustment, or a combination of the two.

In this chapter I have briefly summarized how the optical and electronic properties of a-Si:H are dependent on the hydrogen content and pointed out that the challenge of uncovering the microscopic details of hydrogen bonding and distribution and their correlation with hydrogen dynamics cannot be answered by standard experimental techniques.

On the other hand, with the continuous improvement of computational capacity and software quality, the simulation of realistic structures is becoming ever more feasible. In particular, Ab Initio Molecular Dynamics (AIMD) allows highly accurate simulation of the dynamical properties of various systems, including amorphous materials. 
The goal of such simulations is to be able to reproduce dynamic processes and follow the diffusion of hydrogen, the bond breaking processes, and the structural reorganization of the material, following external perturbations. The DB creation process in tritiated amorphous silicon can provide a simple and convenient source of experimental data that can be used as a basis for such simulations, since the tritium decay process is well understood, and its effect on a-Si:H can be treated as the simple removal of an hydrogen atom from an existing $\mathrm{Si}-\mathrm{H}$ bond.

The main challenge is of course to make sure that the simulated structure is indeed a realistic one. The author of this chapter has shown that several models lack the necessary realism, since the validation of the model is based on the radial distribution function of the $\mathrm{Si}-\mathrm{Si}$ bonds. The author has also shown that the reproduction of the vibrational modes of a$\mathrm{Si}: \mathrm{H}$ represents a much better validation test for a realistic structure. As the continuous advances in computational science will allow for the use of bigger simulated structures, the future direction of these studies should aim at reproducing other fundamental properties, such as the band-gap, the density of states, etc. By achieving this goal, it will be possible then to simulate dynamic processes too, such as the SW effect, and to shed light both on the formation phase of the dangling bonds and on the curing phase.

\section{Acknowledgment}

The work by the author was supported by the Shared Hierarchical Academic Research Computing Network (SHARCNET) and Natural Sciences and Engineering Research Council of Canada (NSERC).

The author would also like to thank Dr. A. Chkrebtii for his invaluable contribution and leadership in the development of the AIMD algorithm. Thanks go also to Dr. J.M. Perz, Dr. S. Zukotynski, and Dr. N. P. Kherani for their support and helpful discussions spread over 20 years.

\section{References}

Abtew, T.A., Drabold, D.A. (2006) Phys. Rev. B, Vol. 74, 085201.

Adler D., (1984) AIP Conference Proceedings, n 120, 70-77.

Akkaya,A., \& Akta,G. (1995). Mater. Lett., Vol. 22, 271.

Baranovski, S. (2006). Charge transport in disordered solids with applications in electronics. John Wiley \$ Sons, ISBN: 9780470095041, New York.

Biswas, R., Li, Q., Pan, B.C., Yoon, Y. (1998) Phys. Rev. B, Vol. 57, 2253.

Biswas, R., Pan, B.C. (2003) Solar Energy Materials and Solar Cells, Vol. 78, 447.

Branz, H.M., Asher, S.E., Nelson, B.P., (1993)Phys. Rev. B Vol. 47, 7061.

Branz, H.M. (1999) Phys. Rev. B Vol. 59, 5498.

Branz, H.M., Asher, S.E., Gleskova, H., Wagner S., (1999)Phys. Rev. B Vol. 59, 5513.

Bruno, G., Capezzuto P., Madan A., (Eds.) (1995) Plasma Deposition of Amorphous SiliconBased Materials. Academic Press, ISBN: 9780121379407, Burlington, MA.

Cheong, H.M., Lee, S.H., Nelson, B.P., Mascarenhas, A., Deb, S.K., (2000) App. Phys. Lett. B Vol. 77, 2686.

Cody, G.D., Tiedje, T., Abeles, B., Brooks B., Goldstein, Y. (1981) Phys. Rev. Lett. Vol. 47, 1480 . 
Costea, S., Gaspari, F., Kosteski, T., Zukotynski, S., Kherani, N. P., Shmayda, W.T. (2000) Mat. Res. Soc. Symp. Proc, Vol. 609, A27.4 (2000).

Costea, S., Pisana, S., Kherani, N.P., Gaspari., F., Kosteski, T., Shmayda, W.T., Zukotynski, S. (2005) Fusion science and technology Vol. 48, 712.

Danesh, P., Pantchev, B., Vlaikova, A. (2005) Nuclear Instruments and Methods in Physics Research B, Vol. 239, 370.

Daouahi, M., Ben Othmane, A., Zellama, K., Zeinert, A., Essamet, M.,Bouchriha, H. (2001) Solid State Communications Vol. 120, 243.

Darwich, R., Roca I. Cabarrocas, P., Vallon, S., Ossikovski, R., Morin, P., Zellama, K. (1995) Phil. Mag. B, Vol. 72, 363.

Dubeau, J., Hamel, L.A., Pochet, T., (1996) Phys. Rev. B 53, 10740

Fehr, M., Schnegg, A., Teutloff, C., Bittl, R., Astakhov, O., Finger, F., Rech, B., Lips, K. (2010) Physica Status Solidi A, Vol. 207, 552.

Gaspari, F., O'Leary, S.K., Zukotynski, S., Perz, J. (1993) J. Non-Cryst. Solids Vol. 155, 149.

Gaspari, F., Kosteski, T., Zukotynski, S., Kherani, N. P., Shmayda, W. (2000) Phil. Mag. B, Vol. 80, 561.

Gaspari, F., Shkrebtii, A., Kupchak, I., Perz, J.M. (2009) Phys. Rev. B Vol 79, 224203.

Gaspari, F., Shkrebtii, A., Kupchak, I., Teatro, T., Ibrahim, Z.A. (2010) 35th IEEE Photovoltaic Specialists Conference Proceedings, Honolulu Hawaii, June 20-25, 003671-75.

Ishimaru, M. (2002) J. Appl. Phys. Vol. 91, 686.

Izumi, S., Hara, S., Kumagai, T., Sakai, S. (2005) J. Cryst. Growth Vol. $274,47$.

Jackson, W.B., Tsai, C.C. (1992) Phys. Rev. B, Vol. 45, 6564.

Jackson, W.B., Santos, P.V., Tsai, C.C. (1993) Phys. Rev. B, Vol. 47, 9993.

Jeffrey, F.R., Shanks, H.R., Danielson, G.C. (1979) Appl. Phys. Lett. Vol. 50, 7034.

Kasap, S. (2005) Principles of Electronic Materials and Devices. McGraw-Hill, retrieved from http:/ / Materials.Usask.Ca.

Kato, S., Aoki, T. (1985) J. Non-Cryst. Solids Vols. 77\&78, 813.

Kherani, N.P., Liu, B., Virk, K., Kosteski, T., Gaspari, F., Shmayda, W.T., Zukotynski, S., Chen, K.P. (2008) J. Appl. Phys. Vol. 103, 024906.

Knights, J.C., Lujan, R.A. (1979) Appl. Phys. Lett. Vol. 35, 244.

Kosteski, T., Gaspari, F., Hum, D., Costea, S., Zukotynski, S., Kherani, N.P., Shmayda, W.T. (2000) Mat. Res. Soc. Symp. Proc. Vol. 609, A30.1.

Kosteski, T., Stradins, P., Kherani, N.P., Gaspari, F., Shmayda, W.T., Sidhu, L., Zukotynski, S. (2003) IEE Proc. Circuits, Devices and Syst., special issue on Amorphous and Microcrystalline Semiconductor Devices, Vol. 150 no. 4, 274.

Kupchak, I. M., Gaspari, F., Shkrebtii, A. I., Perz, J. M. (2008) J. Appl. Phys. Vol. 104, 123525-1

Laaziri, K., Kycia, S., Roorda, S., Chicoine, M. Robertson, J. L., Wang, J., Moss, S. C. (1999) Phys. Rev. Lett. Vol. 82, 3460.

Ley, L. (1983) "Photoemission and Optical properties", in The Physics of Hydrogenated Amorphous Silicon, Vol II, Eds. J.D. Joannopoulos \& G. Lucovski, Springer-Vderlag, ISBN: 0387128077, New York.।

Longeaud, C., Roy, D., Teukam Hangouan, Z. (2000) App. Phys. Lett. Vol. 77, 3604.

Lucovski, G., Davidson, B.N., Parsons, G.N., Wang, C. (1989) J. Non-Cryst. Solids Vol. 114, 154.

Malik, S. M., O'Leary, S. K. (2004) J. Non Cryst. Solids, Vol. 336, 64. 
Morigaki, K., Hikita, H. (2007) Phys. Rev. B 76, 085201

Morigaki, K., Takeda, K., Hikita, H., Ogihara, C., Roca i Cabarrocas, P. (2008) J. Non-Cryst. Solids, Vol. 354, 2131.

Mott, N. (1983) "Conductivity, Localization, and the Mobility Edge", in The Physics of Hydrogenated Amorphous Silicon, Vol II, Eds. J.D. Joannopoulos \& G. Lucovski, Springer-Verlag, ISBN: 0387128077, New York.

O'Leary, S.K., Sidhu, L.S., Zukotynski, S., Perz, J.M. (1996) Canadian Journal of Physics, Vol. 74, S256-9.

Powell, M.J., Deane, S.C., (1996) Phys. Rev. B, Vol. 53, 10121.

Rui, Y., Mei, J., Xu, J., Yang, L., Li, W., Chen, K. (2005) Proceedings of SPIE - The International Society for Optical Engineering, Vol. 5774, 279.

Santos, P.V., Johnson, M.N., Street, R.A. (1991) Phys. Rev. Lett. Vol. 67, 2686.

Santos, P.V., Johnson, N.M., Street, R.A., (1992) Mat. Res. Symp. Proc. Vol. 258, 353.

Santos, P.V., Johnson, M.N., Street, R.A. (1993) J. Non-Cryst. Solids Vols. 164-166, Part I, 277.

Schneider, U., Schröder, B. (1990) Photovoltaic Specialists Conference. Conference Record of the Twenty First IEEE, vol. 2, 1521.

Searle, T. (Ed.) (1998) Amorphous Silicon and its Alloys, INSPEC, ISBN: 0852969228, London.

Sholz, A., Schröder, B., Oechsner, H. (1994) Mat. Res. Symp. Proc. Vol. 336, 293.

Sidhu, L. S., Kosteski, T., Zukotynski, S., Kherani, N. P. (1999) J. Appl. Phys. Vol. 85, 2574.

Singh, R., Prakash, S., Shukla, N., Prasad, R. (2004) Phys. Rev. B Vol. 70, 115213.

Smets, A.H.M., van de Sanden, M.C.M. (2007) Phys. Rev. B, Vol. 76, 073202.

Staebler, D.L., Wronski, C.R. (1977) Appl. Phys. Lett. Vol. 31, 292.

Street, R.A., Biegelsen, D., Stuke, J., (1979) Philos. Mag. B Vol. 40, 451.

Street, R.A. (1980) Phys. Rev. B, Vol. 21, 5775.

Street, R.A. (1991) Hydrogenated Amorphous Silicon, Cambridge University Press, ISBN: 0521371562, New York.

Street, R.A. (Ed.) (2000) Technology and Applications of Amorphous Silicon, Springer Verlag, ISBN: 3540657142, New York.

Street, R.A., Tsai, C.C. (1988) Philos. Mag. Vol. B57, 663.

Stutzmann M., Jackson W.B., Tsai, C.C. (1985), Phys. Rev. B, Vol. 32, n 1, 23-47

Stutzmann M., (1991) in Amorphous and Microcrystalline Amorphous Devices, Vol. II, Ed. J. Kanicki, Atech House, Boston, p. 129.

Tauc, J., Grigorovici, R., Vancu, A. (1966) Phys. Status Solidi, Vol. 15, 627.

Thevaril, J.J., O'Leary, S.K. (2010) J. Appl. Phys., Vol. 107, 083105.

Tuttle, B., Adams, J. B. (1997) Phys. Rev. B Vol. 56, 4565.

Ukpong, A.M. ((2007) Turkish Journal of Physics, Vol. 31, 317.

Van de Walle, C.G., Street, R.A. (1994) Phys. Rev. B, Vol. 49, n 20, 14766-9.

Van de Walle, C.G., Street, R.A. (1995) Mat. Res. Soc. Symp. Proc., Vol. 377, 389.

Yelon, A., Fritzsche, H, Branz, H.M., (2000) J. Non-Cryst. Sol. Vols. 266-268, 437.

Ju, T., Whitaker, J., Zukotynski, S., Kherani, N., Taylor, P.C., Stradins, P. (2007) Mat. Res. Soc. Symp. Proc. Vol. 989, 9.

Whitaker J., Viner, J., Zukotynski, S., Johnson, E., Taylor, P.C., Stradins, P. (2004) Mat. Res. Soc. Symp. Proc. Vol. 808, 153.

Zanzucchi, P.J., Wronski, C.R., Carlson, D.E. (1977) J. Appl. Phys. Vol. 48, 5227. 
Zeman, M. (2006) “Advanced Amorphous Silicon Solar Cell Technologies”, in Thin Film Solar Cells: Fabrication, Characterization and Applications, Eds. J. Poortmans \& V. Arkhipov, John Wiley \& Sons, New York.

Zhang, S.B., Branz, H.M., (2001) Phys. Rev. Lett. Vol. 87, 105503

Zukotynski, S., Gaspari, F., Kherani, N., Kosteski, T., Law, K., Shmayda, W.T., Tan, C.M. (2002) J. Non-Cryst. Solids Vols. 299-302, 476. 


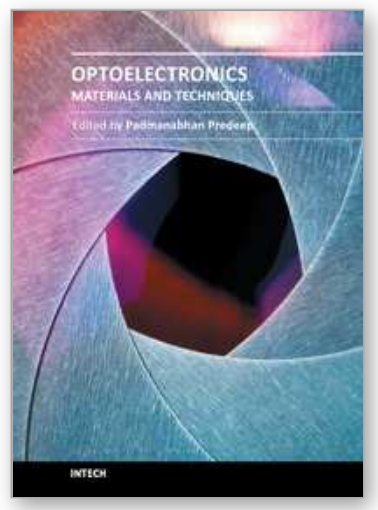

\author{
Optoelectronics - Materials and Techniques \\ Edited by Prof. P. Predeep
}

ISBN 978-953-307-276-0

Hard cover, 484 pages

Publisher InTech

Published online 26, September, 2011

Published in print edition September, 2011

Optoelectronics - Materials and Techniques is the first part of an edited anthology on the multifaceted areas of optoelectronics by a selected group of authors including promising novices to the experts in the field.

Photonics and optoelectronics are making an impact multiple times the semiconductor revolution made on the quality of our life. In telecommunication, entertainment devices, computational techniques, clean energy harvesting, medical instrumentation, materials and device characterization and scores of other areas of R\&D the science of optics and electronics get coupled by fine technology advances to make incredibly large strides. The technology of light has advanced to a stage where disciplines sans boundaries are finding it indispensable. Smart materials and devices are fast emerging and being tested and applications developed in an unimaginable pace and speed. Here has been made an attempt to capture some of the materials and techniques and underlying physical and technical phenomena that make such developments possible through some real time players in the field contributing their work and this is sure to make this collection of essays extremely useful to students and other stake holders such as researchers and materials scientists in the area of optoelectronics.

\title{
How to reference
}

In order to correctly reference this scholarly work, feel free to copy and paste the following:

Franco Gaspari (2011). Optoelectronic Properties of Amorphous Silicon the Role of Hydrogen: from Experiment to Modeling, Optoelectronics - Materials and Techniques, Prof. P. Predeep (Ed.), ISBN: 978-953307-276-0, InTech, Available from: http://www.intechopen.com/books/optoelectronics-materials-andtechniques/optoelectronic-properties-of-amorphous-silicon-the-role-of-hydrogen-from-experiment-to-modeling

\section{INTECH}

open science | open minds

\section{InTech Europe}

University Campus STeP Ri

Slavka Krautzeka 83/A

51000 Rijeka, Croatia

Phone: +385 (51) 770447

Fax: +385 (51) 686166

www.intechopen.com

\section{InTech China}

Unit 405, Office Block, Hotel Equatorial Shanghai

No.65, Yan An Road (West), Shanghai, 200040, China

中国上海市延安西路 65 号上海国际贵都大饭店办公楼 405 单元

Phone: +86-21-62489820

Fax: $+86-21-62489821$ 
(C) 2011 The Author(s). Licensee IntechOpen. This chapter is distributed under the terms of the Creative Commons Attribution-NonCommercialShareAlike-3.0 License, which permits use, distribution and reproduction for non-commercial purposes, provided the original is properly cited and derivative works building on this content are distributed under the same license. 